\title{
Homolactic fermentation from glucose and cellobiose using Bacillus subtilis
}

\author{
Susana Romero-Garciaํ, Claudia Hernández-Bustos ${ }^{1}$, Enrique Merino², \\ Guillermo Gosset ${ }^{1}$ and Alfredo Martinez*1
}

Address: ${ }^{1}$ Departamento de Ingeniería Celular y Biocatálisis, Instituto de Biotecnología, Universidad Nacional Autónoma de México, A.P. 510-3 Cuernavaca, Mor. 62250, México and ²Departamento de Microbiología Molecular, Instituto de Biotecnología, Universidad Nacional Autónoma de México. Cuernavaca, Morelos, México

Email: Susana Romero-Garcia - sugar_cia@yahoo.com; Claudia Hernández-Bustos - ibeth_889@hotmail.com;

Enrique Merino - merino@ibt.unam.mx; Guillermo Gosset - gosset@ibt.unam.mx; Alfredo Martinez* - alfredo@ibt.unam.mx

* Corresponding author

Published: 21 April 2009

Microbial Cell Factories 2009, 8:23 doi:10.1 186/1475-2859-8-23
Received: 20 February 2009

Accepted: 21 April 2009

This article is available from: http://www.microbialcellfactories.com/content/8/I/23

(c) 2009 Romero-Garcia et al; licensee BioMed Central Ltd.

This is an Open Access article distributed under the terms of the Creative Commons Attribution License (http://creativecommons.org/licenses/by/2.0), which permits unrestricted use, distribution, and reproduction in any medium, provided the original work is properly cited.

\begin{abstract}
Backgroung: Biodegradable plastics can be made from polylactate, which is a polymer made from lactic acid. This compound can be produced from renewable resources as substrates using microorganisms. Bacillus subtilis is a Gram-positive bacterium recognized as a GRAS microorganism (generally regarded as safe) by the FDA. B. subtilis produces and secretes different kind of enzymes, such as proteases, cellulases, xylanases and amylases to utilize carbon sources more complex than the monosaccharides present in the environment. Thus, $B$. subtilis could be potentially used to hydrolyze carbohydrate polymers contained in lignocellulosic biomass to produce chemical commodities. Enzymatic hydrolysis of the cellulosic fraction of agroindustrial wastes produces cellobiose and a lower amount of glucose. Under aerobic conditions, B. subtilis grows using cellobiose as substrate.
\end{abstract}

Results: In this study, we proved that under non-aerated conditions, B. subtilis ferments cellobiose to produce L-lactate with $82 \%$ of the theoretical yield, and with a specific rate of L-lactate production similar to that one obtained fermenting glucose. Under fermentative conditions in a complex media supplemented with glucose, B. subtilis produces L-lactate and a low amount of 2,3butanediol. To increase the L-lactate production of this organism, we generated the $B$ subtilis $\mathrm{CHI}$ alsS- strain that lacks the ability to synthesize 2,3-butanediol. Inactivation of this pathway, that competed for pyruvate availability, let a $15 \%$ increase in L-lactate yield from glucose compared with the parental strain. $\mathrm{CHI}$ alsS-fermented 5 and $10 \%$ of glucose to completion in mineral medium supplemented with yeast extract in four and nine days, respectively. $\mathrm{CHI}$ als $\mathrm{S}^{-}$produced $105 \mathrm{~g} / \mathrm{L}$ of L-lactate in this last medium supplemented with $10 \%$ of glucose. The L-lactate yield was up to $95 \%$ using mineral media, and the optical purity of L-lactate was of $99.5 \%$ since B. subtilis has only one gene (IctE) that exclusively encodes a L-lactate deshydrogenase.

Conclusion: This study shows that by taking advantage of the cellobiose utilization capability and osmotic stress high resistance of $B$. subtilis, a robust process for L-lactate production can be developed. 


\section{Background}

The chemical industry has come under increasing pressure to make chemical production more eco-friendly and independent from fossil resources. The development of industrial processes based on microorganisms can importantly help to eliminate the use or generation of hazardous substances and can support the shift from the actual fossil resources dependence toward sustainable and eco-safety industrial processes [1]. One example of this eco-safety process can be found in the production of biodegradable plastics from lactic acid that involves polylactate synthesis. Physical properties and biodegradation rates of polylactate can vary depending on the $\mathrm{D}$ - and L-enantiomers blend [2]. In addition, lactic acid production using microorganisms eliminates the hazard substances generation and the D- and L-enantiomers can be produced in an optically pure state.

Ninety percent of current commercial lactic acid (about 72,000 ton/year) is produced from mono- and disaccharides by culture fermentation using lactic acid bacteria. It is also possible to use other renewable resources such as agroindustrial wastes as substrates to produce lactic acid, although a pretreatment to liberate simple sugars is needed [3]. Cellulose is hydrolyzed by a combination of cellobiohydrolase and endoglucanase activities with cellobiose as the primary product. The ability to utilize cellobiose is widespread among gram-positive, gram-negative and Archeal genera [4].

A variety of microorganisms that produce L-lactate, such as Escherichia coli [5,6], Saccharomyces cerevisiae [7], Lactobacillus sp [8,9], and lactic acid bacteria [3], have been developed for increasing their L-lactate titer, rising their Llactate specific and volumetric productivity, rising their substrate range to include pentose sugars and disaccharides, and eliminating some of their growth requirements. Nevertheless, new biocatalysts are needed to use alternative bio-based feedstocks, like agroindustrial wastes, thus avoiding the use of fungi cellulases, exhibiting high L-lactate yields with high quiral purity.

Bacillus subtilis is a Gram-positive bacterium, which is a GRAS microorganism (generally regarded as safe) by the FDA. B. subtilis can be grown using cellobiose under aerobic conditions [10], because it has the celRABCD operon which encodes the EII permease and phospho- $\beta$-glucosidase proteins [4]. Under non-aerated conditions, B. subtilis produces L-lactate and 2,3-butanediol from glucose [11].

In this paper, we report the L-lactate synthesis from cellobiose during non-aerated cultures using $B$. subtilis. In addition, we eliminated the 2,3-butanediol production of $B$. subtilis (strain CH1 alsS-) increasing L-lactate yield by $15 \%$ compared to parental strain. $\mathrm{CH} 1$ alsS-fermented 5 and $10 \%$ of glucose completely producing just optical pure Llactate in mineral medium supplemented with yeast extract.

\section{Results and discussion \\ B. subtilis produces L-lactate from cellobiose under fermentative conditions}

B. subtilis $\mathrm{CH} 1$ is a prototroph strain that was constructed to avoid the auxotrophic requirements of $B$. subtilis WB700 (see Methods section). To determine the ability of B. subtilis $\mathrm{CH} 1$ to grow using cellobiose under non-aerated conditions, the characterization of this strain using LB (Luria Bertani) and MM (Mineral Media) supplemented with cellobiose $(10 \mathrm{~g} / \mathrm{L})$ was undertaken (Figs. $1 \mathrm{~A}$ and 2A). Also, B. subtilis CH1 was grown in LB and MM supplemented with glucose $(10 \mathrm{~g} / \mathrm{L})$ as control (Figs. $1 \mathrm{~B}$ and 2B).

B. subtilis $\mathrm{CH} 1$ grew exponentially during the first $12 \mathrm{~h}$ using cellobiose or glucose in LB medium (Fig. 1A and 1B), although the specific growth rate of strain CH1 in LB medium using cellobiose diminished 2-fold in comparison with glucose (Table 1), and the maximum biomass

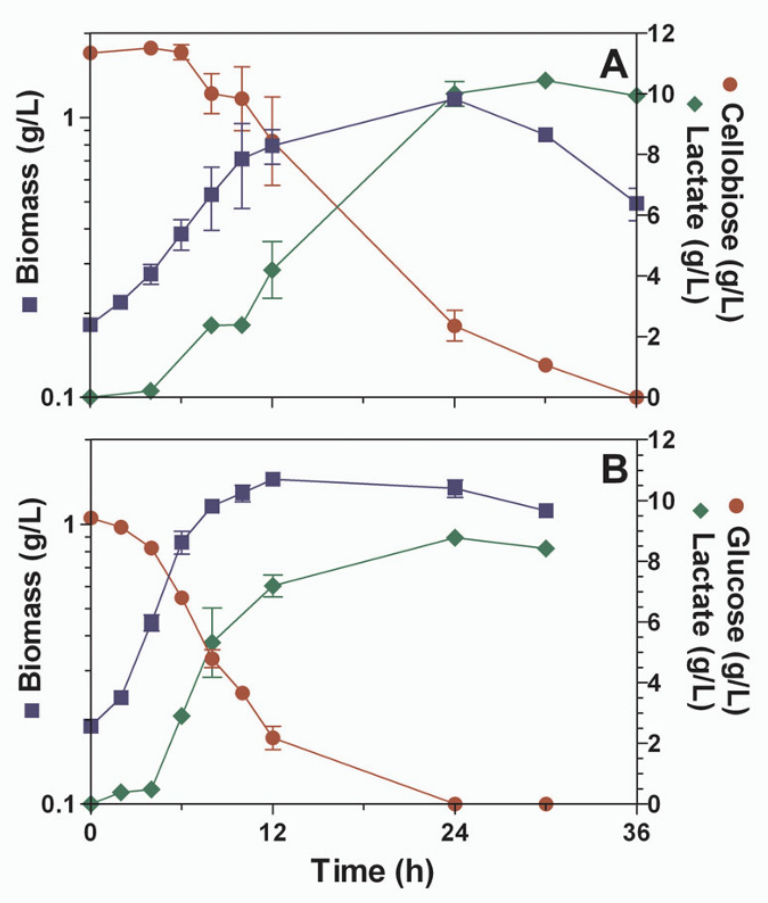

Figure I

Characterization of Bacillus subtilis CHI in LB broth supplemented with 10 g/L of sugar under non-aerated conditions. (A) Cellobiose fermentation, (B) Glucose fermentation. 


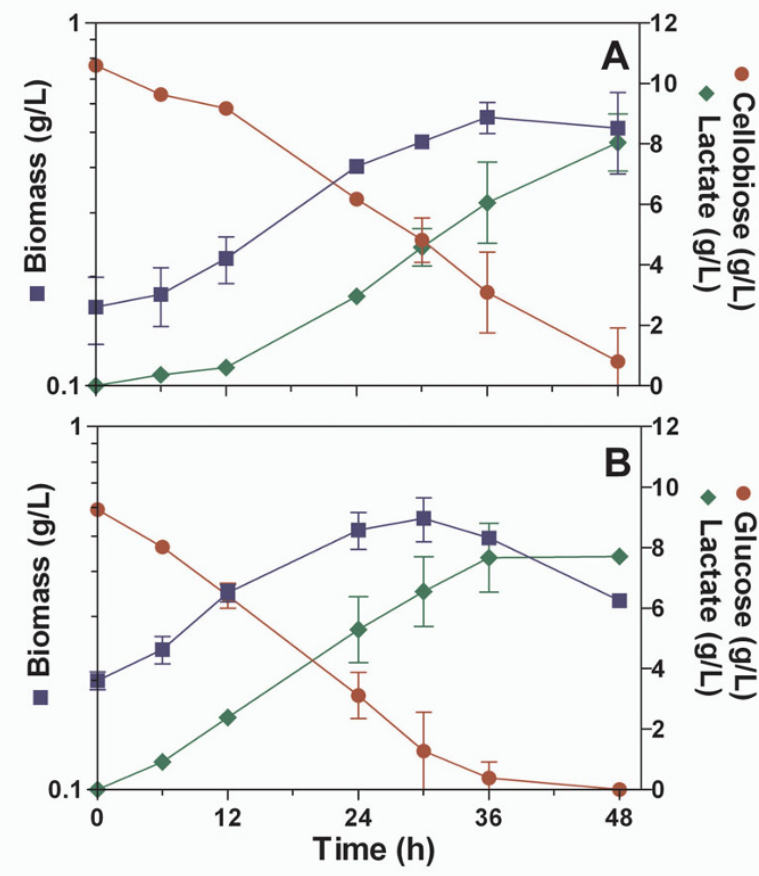

Figure 2

Characterization of Bacillus subtilis $\mathrm{CHI}$ in Mineral Medium supplemented with $10 \mathrm{~g} / \mathrm{L}$ of sugar under non-aerated conditions. (A) Cellobiose fermentation, (B) Glucose fermentation.

obtained at the end of exponential growth was similar using both sugars (Table 1 ). The specific rate of cellobiose consumption of strain $\mathrm{CH} 1$ in LB medium diminished $50 \%$ in comparison with glucose; however, the specific rates of cellobiose and glucose consumption were similar in MM (Table 1).

The cellobiose consumption in LB medium for strain $\mathrm{CH} 1$ was delayed $6 \mathrm{~h}$, probably due to a slight inhibition of cellobiose utilization by some media components. We suggest that during this time strain $\mathrm{CH} 1$ used these media components to grow and to produce L-lactate. Because B. subtilis could produce L-lactate from media components and from cellobiose, the L-lactate yield was higher than $100 \%$ of the theoretical if it is considered only the cellobiose cosumption (Fig. 1A and Table 2). Cellobiose utilization in MM was not delayed, supporting the hypothesis that there was inhibition by LB components (Fig. 2A).

B. subtilis $\mathrm{CH} 1$ fermented cellobiose and glucose using mineral or complex medium, and produced L-lactate as main fermentation product. In all the culture fermentations, the L-lactate production specific rates were larger in the exponential than in the stationary phase (Table 2). The L-lactate production specific rates were similar, using cellobiose or glucose, in complex or mineral media during the stationary phase (Table 2).

Strain CH1 consumed cellobiose $(10 \mathrm{~g} / \mathrm{L})$ in both LB and MM completely (Figs. 1A and 2A), and produced L-lactate with a yield of $82 \%$ of the theoretical in MM (Table 2).

It is known that some Lactobacillus spp use cellobiose as carbon source, but there is little information about lactic acid production from cellobiose. L. delbrueckii had obtained better yields and higher quantities of L-lactate from glucose in comparison with several lactic acid bacteria [3]. Recently, Adsul et al, reported a L. delbrueckii mutant that produced $90 \mathrm{~g} / \mathrm{L}$ of lactic acid from $100 \mathrm{~g} / \mathrm{L}$ of cellobiose [9]; however, they do not mention the optical purity of the product. The principal problem with lactic acid production in Lactobacillus spp has been the optical purity of the product which is not higher than 95\% because some lactic acid bacteria have both, $\mathrm{L}$ - and D-lactate dehydrogenases $[3,7]$, and the $5 \%$ of chiral impurity of the L-lactate may increase the cost associated with purification, which is very complex and expensive to solve.

Although the L-lactate yield and productivity of $L$. delbrueckii mutant are better than those from the strain tested in this report, the optical purity of the L-lactate produced by B. subtilis $\mathrm{CH} 1$ was of $99.5 \%$, tested with an enzymatic

Table I: Kinetic parameters for batch cultures of B. subtilis CHI grown in LB or mineral medium supplemented with glucose or cellobiose (10 g/L).

\begin{tabular}{|c|c|c|c|c|c|c|}
\hline Medium & Sugar & $\begin{array}{c}{ }^{a} X_{\text {MAX }} \\
(g / L)\end{array}$ & $\underset{\left(h^{-1}\right)}{\mu}$ & $\begin{array}{c}{ }^{b Y_{X / S}} \\
\left(g_{D C W} g_{S}\right)\end{array}$ & $\begin{array}{c}{ }^{\mathrm{c}} \mathrm{q}_{\mathrm{S}} \\
\left(\mathrm{g}_{\mathrm{S}} / \mathrm{g}_{\mathrm{DCW}}\right.\end{array}$ & ${ }^{\mathrm{d} C o n s u m e d ~ s u g a r}(\mathrm{~g} / \mathrm{L})$ \\
\hline \multirow[t]{2}{*}{ Luria } & Glucose & 0.67 & 0.30 & 0.26 & 1.17 & 2.6 \\
\hline & Cellobiose & 0.61 & 0.13 & 0.23 & 0.60 & 2.9 \\
\hline \multirow[t]{2}{*}{ Mineral } & Glucose & 0.32 & 0.04 & 0.05 & 0.77 & 6.1 \\
\hline & Cellobiose & 0.30 & 0.04 & 0.06 & 0.66 & 4.8 \\
\hline
\end{tabular}

aMaximum biomass obtained at the end of the exponential growth.

bBiomass yield from substrate during the exponential growth.

cSpecific rate of sugar consumption during the exponential phase.

dConsumed sugar during the exponential growth. 
Table 2: Kinetic parameters for L-lactate production for batch cultures of B subtilis CHI grown in LB or mineral medium supplemented with glucose or cellobiose $(10 \mathrm{~g} / \mathrm{L})$.

\begin{tabular}{|c|c|c|c|c|c|c|}
\hline Medium & Sugar & ${ }^{a} Y_{P / S}\left(g_{P} / g_{S}\right)$ & ${ }^{b} q_{P}\left(g_{P} / g_{D C W} * h\right)$ & ${ }^{c} \mathrm{q}_{\mathrm{P}}\left(\mathrm{g}_{\mathrm{P}} / \mathrm{g}_{\mathrm{DCW}} * \mathrm{~h}\right)$ & $\begin{array}{c}{ }^{d} Q_{p} \\
\left(g_{p} / L^{*} h\right)\end{array}$ & eFinal L-lactate $(\mathrm{g} / \mathrm{L})$ \\
\hline \multirow[t]{2}{*}{ Luria } & Glucose & 1.23 & 2.25 & 0.28 & 0.48 & 11.6 \\
\hline & Cellobiose & 1.14 & 1.69 & 0.26 & 0.36 & 13.0 \\
\hline \multirow[t]{2}{*}{ Mineral } & Glucose & 0.83 & 0.66 & 0.21 & 0.16 & 7.7 \\
\hline & Cellobiose & 0.82 & 0.58 & 0.38 & 0.17 & 8.1 \\
\hline
\end{tabular}

aL-lactate yield from sugar at the end of fermentation.

bSpecific rate of L-lactate production during the exponential growth.

'Specific rate of L-lactate production during the stationary phase.

dVolumetric productivity of L-lactate.

eL-lactate produced at the end of the culture.

assay that detects and quantifies exclusively the L- enantiomer of lactate. This value was compared with the total quantity of lactate measured by HPLC. Then B. subtilis represents an alternative to obtain optical pure L-lactate from other sugar like arabinose and polymers like cellulose. To our knowledge, there are no previous reports on cellobiose utilization producing L-lactate under fermentative conditions using B. subtilis.

\section{B. subtilis biomass increased when $M M$ was supplemented with yeast extract or corn steep liquor}

The growth of $B$. subtilis in mineral medium (MM) diminished compared to complex medium like LB (Table 1). To increase both, B. subtilis growth and L-lactate production specific rate on mineral medium, this medium was supplemented with increased quantities of yeast extract industrial grade (YE) or corn steep liquor (CSL); components that are rich in vitamins and minerals and are not expensive.

Specific growth rate of $B$. subtilis $\mathrm{CH} 1$ increased 6-fold or 8 -fold when $\mathrm{MM}$ was supplemented with $8 \mathrm{~g} / \mathrm{L}$ of $\mathrm{YE}$ or with $10 \mathrm{~g} / \mathrm{L}$ of CSL, respectively. The addition of quantities larger than $8 \mathrm{~g} / \mathrm{L}$ of YE or $10 \mathrm{~g} / \mathrm{L}$ of CSL to mineral medium did not increase the specific growth rate of $B$. subtilis $\mathrm{CH} 1$, although, biomass obtained at the end of exponential phase increased in proportion with the addition of increasing quantities of YE or CSL (data not shown).

In MM supplemented with $8 \mathrm{~g} / \mathrm{L}$ of $\mathrm{YE}$, L-lactate yield diminished $8 \%$ in comparison with MM medium. In MM supplemented with $10 \mathrm{~g} / \mathrm{L}$ of $\mathrm{CSL}$, L-lactate yield increased $6 \%$ in comparison with MM medium, but CSL had small quantities of D-lactate obtaining in the batch culture a racemic mixture of $\mathrm{L}$ - and D-lactate.

The L-lactate production specific rates of $\mathrm{CH} 1$ during exponential phase of $B$. subtilis in MM supplemented with YE or CSL diminished $45 \%$ in comparison to LB, although
L-lactate production specific rates during stationary phase were similar in LB or MM supplemented with YE or CSL (data not shown). Thus, MM supplemented with $8 \mathrm{~g} / \mathrm{L}$ of yeast extract was chosen to assess the capability of L-lactate production.

\section{L-lactate yield and L-lactate production specific rate increased when butanediol synthesis was suppressed}

Strain $\mathrm{CH} 1$ als $S^{-}$was constructed to eliminate pyruvate competition between the L-lactate and the 2,3-butanediol biosynthetic pathways. In strain $\mathrm{CH} 1$ als $S$, the butanediol production was eliminated by interrupting the gene that encodes the acetolactate synthase enzyme (ALSS), which is the first enzyme of the pathway.

Strain $\mathrm{CH} 1$ alsS- was characterized under non-aerated conditions using LB medium supplemented with $20 \mathrm{~g} / \mathrm{L}$ of glucose, and strain $\mathrm{CH} 1$ was included as a control (Table 3 ). The elimination of the butanediol pathway in strain $\mathrm{CH} 1$ alsS-affected, neither the growth specific rate, nor the maximum biomass at the end of exponential phase (Table $3)$.

During the exponential growth, the glucose consumption specific rate of $\mathrm{CH} 1$ alsS- increased $12 \%$ compared to $\mathrm{CH} 1$; this behavior probably caused a $15 \%$ increase in Llactate production specific rate of $\mathrm{CH} 1$ alsS- compared to the control (Table 3). During the stationary phase, the elimination of the butanediol pathway in strain $\mathrm{CH} 1 \mathrm{als} S$ caused more carbon to be directed to L-lactate production, thus increasing by $20 \%$ the L-lactate production specific rate of $\mathrm{CH} 1$ alsS-compared to $\mathrm{CH} 1$ (Table 3 ).

L-lactate volumetric productivity of $\mathrm{CH} 1$ als $\mathrm{S}$ - increased $15 \%$ compared with the control. L-lactate yield of $\mathrm{CH} 1$ als $S$ - increased $15 \%$ up of the theoretical considering just the consumed glucose, this result suggests that $\mathrm{CH} 1$ als $\mathrm{S}$ produced L-lactate from glucose and components media (data not shown). 
Table 3: Kinetic parameters for batch cultures of B. subtilis $\mathrm{CHI}$ and $\mathrm{CHI}$ alsS- grown in LB medium supplemented with glucose (20 g/L).

\begin{tabular}{|c|c|c|c|c|c|c|c|}
\hline B. subtilis strain & $\begin{array}{l}{ }^{a} X_{\text {MAX }} \\
(g / L)\end{array}$ & $\underset{\left(h^{-1}\right)}{\mu}$ & $\left(g_{s} / g_{D C W}{ }^{b}{ }^{\prime} h\right)$ & 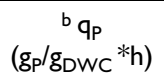 & $\left(g_{S} / g_{D C W}{ }^{c} q_{S}\right)$ & $\left(g_{P} / g_{D C W} q_{P} * h\right)$ & $\begin{array}{c}\text { Butanediol } \\
(g / L)\end{array}$ \\
\hline $\mathrm{CHI}$ & 0.66 & 0.34 & 1.79 & 2.29 & 0.52 & 0.47 & 6 \\
\hline $\mathrm{CHI}$ alss- & 0.62 & 0.32 & 1.99 & 2.63 & 0.51 & 0.56 & 0 \\
\hline
\end{tabular}

a Maximum biomass obtained at the end of exponential growth.

$q_{s}$ : specific rate of sugar consumption.

$\mathrm{q}_{\mathrm{p}}$ : Specific rate of L-lactate production

$b$ During the exponential growth.

c During the stationary growth.

The results presented above show that the butanediol production of strain $\mathrm{CH} 1$ alsS- was completely abolished, this strain produced only L-lactate in LB with high yield, productivity, and optically pure state, thus eliminating the need for purification from a racemic mixture.

\section{B. subtilis $\mathrm{CHI}$ alsS- fermented to completion $5 \%$ and $10 \%$ glucose, and produced L-lactate as the exclusive fermentation product}

To evaluate the ability of L-lactate production of $B$. subtilis $\mathrm{CH} 1$ als $S^{-}$, this strain was challenged to ferment to completion 5 and $10 \%$ of glucose, just as the same high sugar concentrations of cellulosic syrups [12]. Strain CH1 alsSwas grown under non-aerated conditions using different media: LB medium, MM, and mineral medium supplemented with $8 \mathrm{~g} / \mathrm{L}$ of yeast extract (MM with YE). All of these media were supplemented with 5 or $10 \%$ of glucose. Biomass formation, glucose consumption and L-lactate production were evaluated (Fig. 3).

When LB was supplemented with $5 \%$ or $10 \%$ of glucose, the specific growth rate of $\mathrm{CH} 1$ alsS- was not affected by the high glucose concentration, possibly due to addition of the non-metabolized protective osmolyte (compare data in Table 3 with Tables 1 and 3). CH1 alsS- grew exponentially during the first $10 \mathrm{~h}$, using glucose and components of the medium. During exponential growth using $\mathrm{LB}$, the specific rate of glucose consumption increased by $10 \%$ when the glucose concentration was augmented from 2 to $5 \%$ or $10 \%$ (Tables 3 and 4 ). Throughout this time, the specific rate of L-lactate production increased 20\% and 55\% when CH1 alsS- grown in LB was supplemented from 2 to $5 \%$ or $10 \%$ of glucose, respectively (Tables 3 and 5). These results suggest that two or more uptake systems are involved in the transport of glucose and that the glucose transport capacity of the cells is not reached at low glucose concentrations. A similar result has been reported for Lactococcus lactis [13].

L-lactate production specific rates were similar during the stationary phase using LB supplemented with $5 \%$ or $10 \%$ of glucose. $\mathrm{CH} 1$ als $S^{-}$consumed all the glucose and pro- duced L-lactate, it also used some components of the rich medium increasing the L-lactate production (Fig. 3E). Although the fermentation with $10 \%$ of glucose in LB was stopped at $174 \mathrm{~h}$ of fermentation elapsed time, $\mathrm{CH} 1$ alsSconsumed $51 \mathrm{~g} / \mathrm{L}$ of glucose and produced only L-lactate (Table 5).

When MM was supplemented with $5 \%$ or $10 \%$ of glucose, the specific growth rate of $\mathrm{CH} 1$ alsS- was similar in both sugar concentrations (Table 4). CH1 alsS- grew exponentially during first $26 \mathrm{~h}$, consuming $3 \mathrm{~g} / \mathrm{L}$ of glucose and producing only L-lactate with yields up to $97 \%$ of the theoretical. During the exponential phase in $\mathrm{MM}$, glucose consumption specific rate of $\mathrm{CH} 1$ alsS-increased $25 \%$
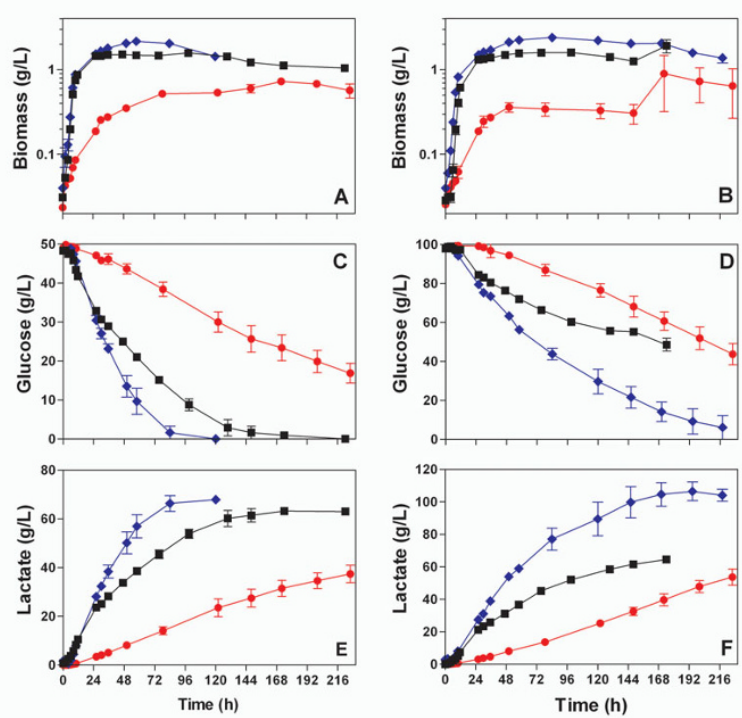

Figure 3

Characterization of $B$. subtilis $\mathrm{CHI}$ alsS- using different media supplemented with 50 g/L (A, C, E) or 100 g/L (B, D, F) of glucose under non-aerated conditions. (A, B) Biomass formation, (C, D) Glucose consumption, and (E, F) L-lactate production using different media: (square) Luria Broth, (diamond) mineral medium supplemented with 8 $\mathrm{g} / \mathrm{L}$ of yeast extract, or (circle) mineral medium. 
Table 4: Kinetic parameters for batch cultures of $B$. subtilis $\mathrm{CHI}$ alsS- grown in LB and mineral medium with 8 g/L of yeast extract (MM with YE) or mineral medium (MM), supplemented with 50 or 100 g/L of glucose (GIc).

\begin{tabular}{|c|c|c|c|c|c|}
\hline Media & $\begin{array}{c}{ }^{a} X_{\text {MAX }} \\
(g / L)\end{array}$ & $\underset{\left(h^{-1}\right)}{\mu}$ & $\begin{array}{c}{ }^{b Y_{X / S}} \\
\left(g_{D C W} / g_{S}\right)\end{array}$ & $\begin{array}{c}{ }^{c} \mathrm{~g}_{\mathrm{s}} / \mathrm{g}_{\mathrm{DCW}} \\
* \mathrm{~h})\end{array}$ & ${ }^{\mathrm{d} C}$ Consumed sugar $(g / L)$ \\
\hline \multicolumn{6}{|l|}{$50 \mathrm{~g} / \mathrm{L} \mathrm{Glc}$} \\
\hline LB & 0.72 & 0.30 & 0.14 & 2.23 & 5.25 \\
\hline MM with YE & 0.83 & 0.27 & 0.16 & 1.74 & 5.26 \\
\hline MM & 0.16 & 0.06 & 0.06 & 1.09 & 2.91 \\
\hline \multicolumn{6}{|l|}{$100 \mathrm{~g} / \mathrm{L} \mathrm{Glc}$} \\
\hline LB & 0.68 & 0.40 & 0.14 & 2.12 & 4.80 \\
\hline MM with YE & 0.78 & 0.29 & 0.09 & 3.45 & 9.26 \\
\hline MM & 0.16 & 0.07 & 0.06 & 1.39 & 3.11 \\
\hline
\end{tabular}

aMaximum biomass obtained at the end of exponential growth.

bBiomass yield from glucose during the exponential growth.

cSpecific rate of glucose consumption during the exponential phase.

dConsumed glucose during the exponential growth.

when the glucose concentration was augmented from 5 to $10 \%$, causing a $5 \%$ increase of the L-lactate specific production rate (Tables 4 and 5).

Throughout the stationary phase using MM, the specific rate of L-lactate production of $\mathrm{CH} 1$ alsS- increased $85 \%$ when glucose concentration was increased from $5 \%$ to $10 \%$ (Table 5), although CH1 alsS- did not finish the glucose in 9 days of elapsed time. The L-lactate yield from glucose diminished from $96 \%$ to $92 \%$ when glucose concentration was increased from 5 to $10 \%$, because $\mathrm{CH} 1$ alsS- produced small quantities of acetic acid in this condition (data not shown). Since the growth rates of the strains studied in this work were low in MM, even though it was supplemented with 1,5 or $10 \%$ of glucose (Tables 1 and 4), the MM was supplemented with $8 \mathrm{~g} / \mathrm{L}$ of yeast extract (YE) besides glucose.
When MM was supplemented with $8 \mathrm{~g} / \mathrm{L}$ of yeast extract (MM with YE) and with $5 \%$ or $10 \%$ of glucose, the specific growth rate of $\mathrm{CH} 1$ als $\mathrm{S}^{-}$was similar in both sugar concentrations (Table 4). $\mathrm{CH} 1$ alsS- grew exponentially during the first $10 \mathrm{~h}$, using glucose and components of the medium. Throughout the exponential growth using MM with YE, the specific rate of glucose consumption had a 2fold increase when glucose concentration was augmented from 5 to $10 \%$, and in consequence the total consumed glucose increased $80 \%$ (Table 4). During exponential growth, the L-lactate specific production rate had a $10 \%$ increase when $\mathrm{CH} 1$ als $S^{-}$grown in LB was supplemented from $5 \%$ to $10 \%$ of glucose (Table 5 ).

During the stationary phase using MM with YE, L-lactate specific production rate of $\mathrm{CH} 1$ alsS- diminished to $30 \%$ when glucose concentration was increased from $5 \%$ to

Table 5: Kinetic parameters for L-lactate production for batch cultures of B subtilis CHI alsS- grown in LB medium and mineral medium with $8 \mathrm{~g} / \mathrm{L}$ of yeast extract (MM with YE) or mineral medium (MM), supplemented with 50 or $100 \mathrm{~g} / \mathrm{L}$ of glucose (Glc).

\begin{tabular}{|c|c|c|c|c|c|}
\hline Media & ${ }^{a} Y_{P / S}\left(g_{P} / g_{S}\right)$ & ${ }^{b} q_{P}\left(g_{P} / g_{D C W} * h\right)$ & $\begin{array}{c}{ }^{{ }^{c} \mathrm{q}_{\mathrm{P}}} \\
\left(\mathrm{g}_{\mathrm{P}} / \mathrm{g}_{\mathrm{DCW}}\right.\end{array}$ & $\begin{array}{c}{ }^{d} Q_{P} \\
\left(g_{P} / L * h\right)\end{array}$ & eFinal L-lactate $(\mathrm{g} / \mathrm{L})$ \\
\hline \multicolumn{6}{|l|}{$50 \mathrm{~g} / \mathrm{L} \mathrm{Glc}$} \\
\hline LB & 1.30 & 3.18 & 0.32 & 0.46 & 63.11 \\
\hline MM with YE & 1.34 & 2.46 & 0.46 & 0.78 & 68.04 \\
\hline MM & 0.96 & 1.28 & 0.36 & 0.17 & 37.40 \\
\hline \multicolumn{6}{|l|}{$100 \mathrm{~g} / \mathrm{L} \mathrm{Glc}$} \\
\hline LB & 1.27 & 4.11 & 0.27 & 0.37 & 64.51 \\
\hline MM with YE & 1.09 & 2.68 & 0.30 & 0.54 & 105.59 \\
\hline MM & 0.92 & 1.33 & 0.67 & 0.24 & 53.69 \\
\hline
\end{tabular}

aL-lactate yield from glucose at the end of fermentation.

bSpecific rate of L-lactate production during the exponential growth.

cSpecific rate of L-lactate production during the stationary phase.

dVolumetric productivity of L-lactate.

eL-lactate produced at the end of the stationary phase. 
$10 \%$. In fact, $\mathrm{CH} 1$ alsS-fermented 5 or $10 \%$ of glucose using MM with YE medium to completion producing exclusively L-lactate with volumetric productivities of 0.78 and 0.54 , respectively (Table 5).

The final L-lactate titer of $105 \mathrm{~g} / \mathrm{L}$ for our strain $\mathrm{CH} 1 \mathrm{alsS}$ compares favorably with that from recombinant bacteria such as L. lactis [14] and E. coli SZ194 [15], and exceeds the performance of previously reported engineered biocatalysts $[5,7,16]$.

\section{Conclusion}

It was found that Bacillus subtilis can consume cellobiose under fermentative conditions and produce L-lactate with high optical purity. We established that a recombinant $B$. subtilis strain was able to produce high yields of L-lactate, using inexpensive mineral medium supplemented with 8 $\mathrm{g} / \mathrm{L}$ of yeast extract. Also, the results in this study show that a robust process for L-lactate production can be developed with $B$. subtilis $\mathrm{CH} 1$ als $S^{-}$, taking advantage of its cellobiose utilization capability and osmotic stress high resistance.

\section{Methods}

\section{Strains and Culture Media}

Bacillus subtilis $\mathrm{CH} 1$ and $\mathrm{CH} 1$ alsS- strains were used in all experiments. B. subtilis $\mathrm{CH} 1$ is a prototroph strain $\left(t r p^{+}\right)$ obtained as described in [11] from B. subtilis WB700 strain (168, trpC2, $\Delta n p r E, \Delta a p r E, \Delta e p r, \Delta b p f, \Delta m p r, \Delta n p r B, \Delta v r p E$, $E r \gamma^{r}, L y n^{r}$ ) [17]. Inactivacion of the alsS gene of $B$. subtilis $\mathrm{CH} 1$ strain to generate strain $\mathrm{CH} 1$ alsS- $(168, \Delta n p r E$, $\Delta a p r E, \Delta e p r, \Delta b p f, \Delta m p r, \Delta n p r B, \Delta v r p E, \Delta a l s S, E r \gamma^{r}, L y n^{r}$, $\left.S p t^{r}\right)$ was performed as described in [11].

Culture media were Luria Bertani (LB) or mineral medium (MM). LB containing (per liter) 5 g yeast extract, $10 \mathrm{~g}$ tryptone, $5 \mathrm{~g} \mathrm{NaCl}$. LB was supplemented with different concentrations of cellobiose or glucose as indicated in Results section. MM [18] containing (per liter) $4 \mathrm{~g}$ $\left(\mathrm{NH}_{4}\right)_{2} \mathrm{SO}_{4}, 5.32 \mathrm{~g} \mathrm{~K}_{2} \mathrm{HPO}_{4}, 6.4 \mathrm{~g} \mathrm{KH}_{2} \mathrm{PO}_{4}, 10 \mathrm{mg}$ citric acid, $0.4 \mathrm{~g} \mathrm{MgSO}_{4} 7 \mathrm{H}_{2} \mathrm{O}, 0.5 \mathrm{mg} \mathrm{MnCl}_{2}, 4 \mathrm{mg} \mathrm{CaCl}{ }_{2}$, and $3 \mathrm{mg} \mathrm{FeSO} 7 \mathrm{H}_{2} \mathrm{O}, \mathrm{pH}$ was adjusted at 7.2 with $\mathrm{NaOH}$. $\mathrm{MM}$ was supplemented with different concentrations of cellobiose or glucose, as indicated in Results section. MM added with glucose was supplemented with different concentrations of yeast extract (YE) or corn steep liquor (CSL), as indicated in Results section. When MM was supplemented with $5 \%$ or $10 \%$ of glucose, $2 \mathrm{mM}$ of betaine (a non-metabolized protective osmolyte) [15] was added.

\section{Inoculum preparation and fermentation conditions}

Strains from frozen vials were plated in Petri dish with solid fermentation media and incubated overnight at $37^{\circ} \mathrm{C}$. Cells from plates were used to inoculate a $250 \mathrm{~mL}$ flask with $150 \mathrm{~mL}$ of the same fermentation media and incubated overnight at $37^{\circ} \mathrm{C}, 120 \mathrm{rpm}$. Erythromycin $(5$ $\mu \mathrm{g} / \mathrm{ml})$ and lyncomycin $(5 \mu \mathrm{g} / \mathrm{ml})$ were added to $\mathrm{CH} 1$ inoculum, and spectinomycin $(100 \mu \mathrm{g} / \mathrm{ml})$ was added to $\mathrm{CH} 1$ als $S^{-}$inoculum. Cells from flasks were centrifuged and utilized as inocula at an initial optical density of 0.1 at $600 \mathrm{~nm}\left(\mathrm{OD}_{600}\right)$. Fermentations were performed in duplicate in minifleaker fermenters [19] containing 200 $\mathrm{mL}$ of fermentation culture media as indicated in Results section. Working conditions were $37^{\circ} \mathrm{C}$, agitation speed of $100 \mathrm{rpm}$, and $\mathrm{pH} 7$ (controlled by automatic additions of 2 or $4 \mathrm{~N} \mathrm{KOH}$ ). Growth was followed by measuring $\mathrm{OD}_{600}$ with a spectrophotometer (Lambda 11, Perking Elmer, Pomona, CA). Supernatants were separated from samples periodically taken and conserved at $-20^{\circ} \mathrm{C}$ for later HPLC analysis.

\section{Analytical methods}

Culture data presented are the average of two independent fermentations. $\mathrm{OD}_{600}$ was converted to dry cellular weight using a standard curve $\left(1 \mathrm{OD}_{600}=0.35 \mathrm{~g} / \mathrm{L}\right.$ of dry cellular weight, DCW). All values of biomass, substrates and products were corrected by dilution factor due to base additions. Glucose and fermentation products in supernatants were measured by HPLC (Model 996, Waters, Millipore Company, Milford, Massachusetts, USA) equipped with a refractive index and an UV detector. Separations were carried out at $50^{\circ} \mathrm{C}$, using an Aminex HPX-87H column (300 $\times 7.8 \mathrm{~mm}$; BioRad), and $5 \mathrm{mM} \mathrm{H}_{2} \mathrm{SO}_{4}$ as mobile phase, at a flow rate of $0.5 \mathrm{~mL} / \mathrm{min}$ (injection volume, $20 \mu \mathrm{l}$ ). L-lactate was measured using a biochemistry analyzer (YSI 2700 SELECT $\left.^{\mathrm{TM}}\right)$, which contains a specific L-lactate oxidase enzyme immobilized in the YSI L-lactate membrane. The purity of L-lactate was evaluated as follows: optical purity $=$ (L-lactate quantity determined in YSI analyzer * 100)/total lactic acid quantity determined in HPLC. In the case of MM supplemented with yeast extract or corn steep liquor, glucose was determined with the biochemistry analyzer using an YSI D-glucose membrane.

\section{Competing interests}

The authors declare that they have no competing interests.

\section{Authors' contributions}

SR-G participated in the design of this study, carried out cloning, construction of the homolactic fermentative strain, fermentations, data analysis and drafted the manuscript. CH-B participated in the construction of the prototroph strain, fermentations and data analysis. EM participated in writing, reviewing and to comment the manuscript. GG participated in writing, reviewing and to comment the manuscript. AM conceived the study, designed and supervised the experiments, participated in results analysis and writing of the manuscript. All authors have read and approved the manuscript. 


\section{Acknowledgements}

We thank to Georgina Hernández for HPLC analysis and to Mercedes Enzaldo, Ricardo Ciria, Alma Valle and Jorge Yáñez for technical support. We thank S.L. Wong (Calgary University, Ca) for kindly providing the $B$. subtilis WB700 strain. This research was supported by the Mexican Council of Science and Technology (CONACyT) grants: CONACyT - SAGARPA 2004-COI-224 and CONACyT - Estado de Morelos MOR-2007-COL80360, and UNAM (PAPIIT-DGAPA: IN220908). Susana Romero was supported by CONACYT scholarship 184798.

\section{References}

I. Hatti-Kaul R, Törnvall U, Gustafsson L, Börjesson P: Industrial biotechnology for the production of bio-based chemicals- a cradle-to-grave perspective. Trends Biotechnol 2007, 25: I I9-I 24.

2. Narayanan N, Roychoudhury PK, Srivastava A: L (+) lactic acid fermentation and its product polymerization. Electronic J Biotechnol 2004, 7(2 167-179 [http://www.ejbiotechnology.info/content/vol7/ issue2/full/7/7.pdf].

3. Hofvendahl K, Hahn-Hägerdal B: Factors affecting the fermentative lactic acid production from renewable resources. Enzyme Microb Technol. 2000, 26(2-4):87-I07.

4. Lai X, Davis FC, Hespell RB, Ingram LO: Cloning of cellobiose phosphoenolpyruvate-dependent phosphotransferase genes: functional expression in recombinant Escherichia coli and identification of a putative binding region for disaccharides. Appl Environ Microbiol I 997, 63(2):355-363.

5. Zhou S, Yomano LP, Shanmugam KT, Ingram LO: Fermentation of I $0 \%$ (w/v) sugar to D(-)-lactate by engineered Escherichia coli B. Biotechnol Lett 2005, 27:189|-1896.

6. Zhou S, Shanmugam KT, Yomano LP, Grabar TB, Ingram LO: Fermentation of $12 \%$ (w/v) glucose to $1.2 \mathrm{M}$ lactate by Escherichia coli strain SZI 94 using mineral salts medium. Biotechnol Lett 2006, 28:663-670.

7. Saitoh S, Ishida N, Onishi T, Tokuhiro K, Nagamori E, Kitamoto K, Takahashi $\mathrm{H}$ : Genetically engineered wine yeast produces a high concentration of L-lactic acid of extremely high optical purity. Appl Environ Microbiol 2005, 7 I(5):2789-2792.

8. Singh SK, Ahmed SU, Pandey A: Metabolic engineering approaches for lactic acid production. Process Biochem 2006, 4l:991-1000.

9. Adsul M, Khire J, Bastawde K, Gokhale D: Production of lactic acid from cellobiose and cellotriose by Lactobacillus delbrueckii mutant Uc-3. Appl Environ Microbiol 2007, 73(I 5):5055-5057.

10. Tobisch S, Glaser P, Krüger S, Hecker M: Identification and characterization of a new $\beta$-glucoside utilization system in Bacillus subtilis. J Bacteriol. 1997, I79(2):496-506.

II. Romero S, Merino E, Gosset G, Martinez A: Metabolic engineering of Bacillus subtilis for ethanol production: lactate dehydrogenase plays a key role in fermentative metabolism. Appl Environ Microbiol 2007, 73(16):5190-5198.

12. Martínez A, Rodríguez ME, Wells ML, York SW, Preston JF, Ingram LO: Detoxification of dilute acid hydrolysates of lignocellulose with lime. Biotechnol Prog 200 I, I 7(2):287-293.

13. Papagianni M, Avramidis N, Filiousis G: Glycolysis and the regulation of glucose transport in Lactococcus lactis spp. lactis in batch and fed-batch culture. Microb Cell Fact 2007, 6:16.

14. Bai DM, Zhao XM, Li XG, Xu SM: Strain improvement and metabolic flux analysis in the wild-type and a mutant Lactobacillus lactis strain for $\mathbf{L}(+)$-lactic acid production. Biotechnol Bioeng 2004, 88:68l-689.

15. Zhou S, Grabar TB, Shanmugam KT, Ingram LO: Betaine tripled the volumetric productivity of $\mathrm{D}(-)$-lactate by Escherichia coli strain SZI32 in mineral salts medium. Biotechnol Lett 2006, 28:67I-676.

16. Skory C: Lactic acid production by Rhizopus oryzae transformants with modified lactate dehydrogenase activity. Appl Microbiol Biotechnol 2004, 64:237-242.

17. Ye RL, Yang LP, Wong SL: Construction of protease deficient Bacillus subtilis strains for expression studies: inactivation of seven extracelluar proteases and the intracellualar LonA protease. Proc International Symposium on Recent Advances in Bioindustry 1996:160-169.
18. Martínez A, Ramírez OT, Valle F: Improvement of culture conditions to overproduce $\beta$-galactosidase from Escherichia coli in Bacillus subtilis. Appl Microbiol Biotechnol 1997, 47( I):40-45.

19. Beall DS, Ohta K, Ingram LO: Parametric studies of ethanol production from xylose and other sugars by recombinant Eschericia coli. Biotechnol Bioeng I991, 38(3):296-303.
Publish with Bio Med Central and every scientist can read your work free of charge

"BioMed Central will be the most significant development for disseminating the results of biomedical research in our lifetime. "

Sir Paul Nurse, Cancer Research UK

Your research papers will be:

- available free of charge to the entire biomedical community

- peer reviewed and published immediately upon acceptance

- cited in PubMed and archived on PubMed Central

- yours - you keep the copyright
BioMedcentral 